\title{
de Sitter gravity from lattice gauge theory
}

\author{
Simon Catterall \\ Department of Physics, Syracuse University, Syracuse, NY13244, USA \\ E-mail: smc@phy.syr.edu \\ Daniel Ferrante \\ Department of Physics, Syracuse University, Syracuse NY13244, USA \\ E-mail: ddferran@syr.edu

\begin{abstract}
Arwen Nicholson
Department of Physics and Astronomy, Edinburgh University, Edinburgh EH9 3JZ, UK

E-mail: arwen.e.nicholson@gmail.com
\end{abstract}

\begin{abstract}
We investigate a lattice model for Euclidean quantum gravity based on discretization of the Palatini formulation of General Relativity. Using Monte Carlo simulation we show that while a naive approach fails to lead to a vacuum state consistent with the emergence of classical spacetime, this problem may be evaded if the lattice action is supplemented by an appropriate counter term. In this new model we find regions of the parameter space which admit a ground state which can be interpreted as (Euclidean) de Sitter space.
\end{abstract}




\section{Contents}

1. Introduction 1

2. Review of the Palatini formalism 2

3. Lattice theory 6

4. Numerical results 8

4.1 Pure Palatini 8

4.2 Palatini plus Wilson 10

5. Discussion

\section{Introduction}

The problem of constructing a theory of gravity which is consistent with quantum mechanics has a long history. The non-renormalizability of quantum general relativity can be traced to the fact that the Newton constant carries the dimensions of length squared corresponding to an action linear in the curvature. Actions containing higher powers of the curvature are usually thought to lead to violations of unitarity and can at best be thought of only as effective field theories [1].

Alternative approaches to gravity have tried to highlight the similarities to gauge theory, where in the case of gravity, the local symmetry corresponds to Lorentz invariance. In this case the corresponding gauge field is called a spin connection. It is necessary to introduce such an object when discussing fermions in curved spacetime where it is partnered by a new field the so-called vierbein which describes the local Lorentz frame and from which the usual metric tensor can be reconstructed. An action coupling the vierbein to the Yang-Mills curvature associated with this spin connection can be written down and shown to reproduce the usual Einstein equations under certain conditions - that the vierbein, regarded as a matrix, be invertible and that the torsion - the antisymmetrized covariant derivative of the vierbein, be zero. It is a first order formulation as both the vierbein and spin connection are to be varied independently to determine the classical equations of motion.

This recasting of gravity in the language of gauge theory is termed the Palatini or Palatinitetrad formulation of General Relativity [2]. In principle this approach gives a natural starting point for a non-perturbative study of quantum gravity since it is possible to discretize the theory while maintaining exact gauge invariance using techniques similar to those employed 
for (lattice) QCD. As such it is complementary to lattice approaches based on Euclidean dynamical triangulations see eg. [3, 4, 5, 6] and causal dynamical triangulations [7, 8, 9] and references therein.

A number of proposals have been made earlier along these lines [10, 11, 12, 13, 14] and numerical studies performed [15, 16, 17]. Unfortunately this previous work encountered several difficulties; chief among these was the observation that the expectation value of the vierbein vanished in the classical weak coupling limit. This condition by itself is sufficient to invalidate the connection between the gauge theory and gravity as we will discuss in the next section.

In this work we have explored the phase structure of a lattice model in which the discrete Palatini action is supplemented by an additional Yang-Mills term. We find a region of the parameter space of the model where the vierbein is non-zero, the torsion small and the curvature constant.

The paper starts with a review of the Palatini formalism in the continuum, and describes its discretization on the lattice. The non-perturbative structure of this theory is then explored using Monte Carlo simulation and we show the classical ground state of the theory corresponds to vanishing vierbein and a curvature which approaches its kinematic limits ${ }^{1}$. Thus the resulting lattice theory does not possess an appropriate ground state which can be identified with a continuum geometry. We then modify the model to incorporate an additional curvature squared operator and survey the expanded phase diagram finding indications of a region where the ground state of theory can be identified with Euclidean de Sitter space. In the final section of the paper we discuss what these results imply and what further work must be done to solidify any possible connection to a theory of gravity.

\section{Review of the Palatini formalism}

The Palatini action can be regarded as the most general action constructed from the curvature of the spin connection and the vierbein which is invariant under local Lorentz transformations and whose definition is independent of the choice of background metric. It takes the form

$$
S_{\text {Palatini }}=\frac{1}{l_{p}^{2}} \int d^{4} x \epsilon_{\mu \nu \lambda \rho} \epsilon^{i j k l} e_{\mu}^{i} e_{\nu}^{j}\left(R_{\lambda \rho}^{k l}-\frac{\Lambda}{6} e_{\lambda}^{k} e_{\rho}^{l}\right)
$$

with $l_{p}=\sqrt{4 \pi G}$ the Planck length. The usual metric of General Relativity is then related to the vierbein via

$$
g_{\mu \nu}=\eta_{i j} e_{\mu}^{i} e_{\nu}^{j}
$$

where $\eta_{i j}=\delta_{i j}$ is a flat (Euclidean) metric which henceforth we shall leave implicit in formulae. This relation is clearly invariant under local $S O(4)$ gauge transformations mediated by

\footnotetext{
${ }^{1}$ In Euclidean space we replace the Lorentz group $S O(3,1)$ by its compact analog $S O(4)$. In the context of the lattice theory this ensures that the local curvature is bounded both above and below
} 
the spin connection $\omega_{\mu}$ where

$$
\omega_{\mu}=\sum_{i<j} \omega_{\mu}^{i j} T^{i j} \quad i<j=1 \ldots 4
$$

with $T^{i j}$ being appropriate generators of $S O(4)$. The curvature $R_{\mu \nu}^{i j}(\omega)$ appearing in eqn. 2.1 is the usual Yang-Mills field strength associated with the spin connection.

$$
R_{\mu \nu}^{i j}=\partial_{\mu} \omega_{\nu}^{i j}-\partial_{\nu} \omega_{\mu}^{i j}+\left[\omega_{\mu}^{i k}, \omega_{\nu}^{k j}\right]
$$

Variation of this action with respect to the spin connection yields the torsion free constraint $D_{[\mu} e_{\nu]}^{i}=0$ which can be used to solve for the spin connection in terms of the vierbein provided the inverse $e_{i}^{\mu}$ exists. The solution is

$$
\omega_{\mu i j}(e)=\frac{1}{2} e_{i}^{\nu} e_{j}^{\rho}\left(\Omega_{\mu \nu \rho}-\Omega_{\nu \rho \mu}+\Omega_{\rho \mu \nu}\right) ;
$$

where,

$$
\Omega_{\mu \nu \rho}=\left(\partial_{\mu} e_{\nu}^{k}-\partial_{\nu} e_{\mu}^{k}\right) e_{\rho k}
$$

Variation of the action with respect to the vierbein then yields the equation,

$$
\epsilon^{i j k l} \epsilon_{\mu \nu \lambda \rho}\left(R_{\mu \nu}^{i j}-\frac{\Lambda}{3} e_{\mu}^{i} e_{\nu}^{j}\right) e_{\lambda}^{k}=0
$$

which, in conjunction with the torsion free solution given above $\omega=\omega_{\mu}(e)$, just reproduces the usual Einstein equation for pure gravity with a cosmological constant. The vacuum solutions are then constant curvature spaces - here Euclidean de Sitter space - the sphere $S^{4}$.

As MacDowell and Mansouri noted [18] it is possible to forge an even stronger connection to Yang-Mills theory by combining the $S O(4)$ spin connection $\omega_{\mu}$ and vierbein $e_{\mu}$ into a single gauge field $A_{\mu}$ associated with a enlarged $S O(5)$ gauge symmetry

$$
A_{\mu}=\omega_{\mu}^{i j} T^{i j}+\frac{1}{l} e_{\mu}^{i} T^{5 i} \quad i, j=1, \ldots 4
$$

where $l$ is some scale inserted to render the vierbein dimensionless. In a similar fashion the $S O(5)$ curvature $F_{\mu \nu}$ can be decomposed into components transforming under the same $S O(4)$ subgroup

$$
\begin{aligned}
F_{\mu \nu} & =\left(R_{\mu \nu}-\frac{1}{l^{2}} e_{[\mu} e_{\nu]}\right)^{i j} T^{i j} \\
& +\frac{1}{l} D_{[\mu} e_{\nu]}^{i} T^{5 i} \quad i, j=1 \ldots 4
\end{aligned}
$$

with $R$ the $S O(4)$ curvature.

An $S O(4)$ invariant action quadratic in this $S O(5)$ curvature may then be written down

$$
S=\kappa \int d^{4} x \epsilon_{\mu \nu \lambda \rho} \epsilon^{i j k l 5} F_{\mu \nu}^{i j} F_{\lambda \rho}^{k l}
$$


By expanding the $S O(5)$ field strengths according to eqn. 2.9 this is easily seen to be nothing more than the Palatini action given in eqn. (2.1) with the dimensionless coupling $\kappa=\frac{1}{2}\left(\frac{l}{l_{p}}\right)^{2}$ giving the size of the Universe in units of the Planck length and $\Lambda={\frac{3}{l^{2}}}^{2}$ Notice that the classical equations of motion derived from the Palatini action just correspond to setting the $S O(5)$ curvature to zero.

This action while formulated in terms of an $S O(5)$ connection exhibits an explicit breaking to $S O(4)$. It is natural to generalize this action slightly to try and realize this as a spontaneous breaking. The following $S O(5)$ action accomplishes this at the expense of introducing an additional scalar field $\phi$.

$$
S=\int d^{4} x \epsilon_{\mu \nu \lambda \rho} \epsilon^{i j k l m} F_{\mu \nu}^{i j} F_{\lambda \rho}^{k l} \phi^{m}
$$

On the assumption that the scalar acquires a vacuum expectation value of the form $\phi^{m}=\kappa \delta^{5 m}$ we recover the previous action.

The appearance of a scalar field $\phi$ and an associated $S O(5)$ gauge symmetry seems at first sight to be somewhat arbitrary. However, it is possible to show that this $S O(5)$ invariant theory arises naturally as a compactification of a Chern-Simons theory in five dimensions. This Chern-Simons theory is a generalization to five dimensions of Witten's formulation of three dimensional gravity as a Chern Simons theory [?, ?]. In the Euclidean case considered here this amounts to constructing a topological gravity theory in five dimensions with internal symmetry group $S O(6)$ and action

$$
S_{\mathrm{CS}}=\int_{M_{5}}<\Omega \wedge \mathcal{R} \wedge \mathcal{R}+\frac{3}{2} \Omega \wedge \Omega \wedge \Omega \wedge \mathcal{R}+\frac{3}{5} \Omega \wedge \Omega \wedge \Omega \wedge \Omega \wedge \Omega>
$$

where for brevity we used the language of differential forms to represent the contraction of spacetime indices with the five dimensional epsilon symbol. The Lie algebra valued connection is

$$
\Omega_{\mu}=\sum_{A<B} \Omega_{\mu}^{A B} J_{A B} ; A, B=1, \ldots, 6 ;
$$

with $J_{A B}=\left[\gamma_{A}, \gamma_{B}\right]$ the generators of $S O(6)$ and $\mathcal{R}$ the curvature. The the angular brackets indicate that the group indices are contracted with the $S O(6)$ invariant tensor $\epsilon_{A B C D E F}$. This Chern-Simons Lagrangian is related to the Euler density in six dimensions via the relation,

$$
d L_{\mathrm{CS}}=\epsilon_{A B C D E F} \mathcal{R}^{A B} \wedge \mathcal{R}^{C D} \wedge \mathcal{R}^{E F}
$$

Stokes' theorem then guarantees that the Chern-Simon's theory is invariant under local gauge transformations up to possible boundary terms.

It is useful at this point to decompose the connection in terms of quantities transforming simply under an $S O(5)$ subgroup

$$
\Omega=\sum_{a<b} A^{a b} J_{a b}+\sum_{a} E^{a} J_{6 a} \quad a, b=1 \ldots 5
$$

\footnotetext{
${ }^{2} \mathrm{~A}$ term quadratic in the $S O(4)$ curvatures also appears $\int \epsilon_{\mu \nu \lambda \rho} \epsilon^{i j k l} R_{\mu \nu}^{i j} R_{\lambda \rho}^{k l}$ but can be neglected as it corresponds to a topological invariant - the Euler number
} 
Similarly the $S O(6)$ field strength decomposes according to

$$
\mathcal{R}=\sum_{a<b}\left(F^{a b}+E^{a} \wedge E^{b}\right) J_{a b}+\sum_{a} D E^{a} J_{6 a}
$$

where $F$ is the $S O(5)$ curvature and $D E$ the torsion. The action up to boundary terms becomes

$$
S_{\mathrm{CS}}=\int_{M_{5}} \epsilon_{a b c d e}\left(E^{a} \wedge F^{b c} \wedge F^{d e}+\frac{2}{3} E^{a} \wedge E^{b} \wedge E^{c} \wedge F^{d e}+\frac{1}{5} E^{a} \wedge E^{b} \wedge E^{c} \wedge E^{d} \wedge E^{e}\right)
$$

In this form it is clear that the action does indeed describe a gravitational theory containing a "Gauss-Bonnet form", an Einstein term and a cosmological constant in five dimensions. Furthermore, this theory is invariant under the full (Euclidean) de Sitter group not just local Lorentz transformations.

To make contact with the MacDowell-Mansouri form of the Palatini action we must compactify this theory down to four dimensions. Let us assume that the five dimensional manifold is of the form $M_{5}=M_{4} \times S^{1} / \mathbb{Z}_{2}$ with $l_{5}$ the extent of the fifth dimension. On the four dimensional boundaries we will assume that the gauge field satisfies the following boundary conditions,

$$
\begin{aligned}
& \Omega_{\mu}=\gamma_{6} \Omega_{\mu} \gamma_{6} ; \mu=1, \ldots, 4 \\
& \Omega_{5}=-\gamma_{6} \Omega_{5} \gamma_{6}
\end{aligned}
$$

This breaks the $S O(6)$ gauge symmetry down to $S O(5)$ at the boundaries with the only surviving four dimensional fields being

$$
E_{5}^{a}, A_{\mu}^{a b}
$$

Returning to the action in eqn. 2.17 it shoul be clear that only the "Gauss-Bonnet" term survives at tree level on the four dimensional boundaries. Indeed, this term becomes

$$
\int d^{4} x \epsilon_{a b c d e} \epsilon_{\mu \nu \lambda \rho} E_{5}^{a} F_{\mu \nu}^{b c} F_{\lambda \rho}^{d e}
$$

This is nothing more than the previous action with the fifth component of the five dimensional vielbein playing the role of the scalar field in the four dimensional theory. The development of a vacuum expectation value for the scalar field could then be realized in terms of the appearance of a non vanishing Polyakov line in the fifth direction extending between the two four dimensional boundaries.

Thus the $\mathrm{SO}(5)$ invariant theory given in eqn. 2.11 can be realized by an appropriate compactification of a topological gravity theory in five dimensions. The possible existence of an underlying exact $S O(5)$ gauge symmetry in the four dimensional theory has some advantages; it allows us to restrict possible counter terms to those invariant under $S O(5)$, it 
makes the choice of the Haar measure for $S O(5)$ natural in the path integral and also, as we now explain, it makes the restoration of diffeomorphism invariance a more likely possibility.

In [?] Witten showed that in fact diffeomorphism invariance is intimately connected to invariance under both lorentz transformations and local translations. In the four dimensional model considered here the result of a general coordinate transformation with parameter $-\xi^{\nu}$ acting on the spin connection and vierbein can be written compactly as a gauge transformation with parameter $\xi^{\mu} A_{\mu}$ acting on the $S O(5)$ gauge field $A=\omega+e$ plus a term which vanishes on flat connections with $F=0$

$$
\delta^{\xi} A_{\mu}=-D_{\mu}\left(\xi^{\nu} A_{\nu}\right)-\xi^{\nu} F_{\mu \nu}
$$

Thus provided the theory is $S O(5)$ invariant and we consider only small fluctuations around a flat background the theory will automatically be invariant under general coordinate transformations.

\section{Lattice theory}

It is straightforward to formulate the Palatini action in the form given by eqn. 2.10 on a hypercubic lattice as was first suggested in [10].

$$
S_{P}=\kappa \sum_{x} \sum_{\mu \nu \rho \lambda} \epsilon_{\mu \nu \lambda \rho} \operatorname{Tr}\left(\gamma_{5} U_{\mu \nu} U_{\lambda \rho}\right)
$$

where

$$
U_{\mu \nu}=U_{\mu}(x) U_{\nu}(x+\mu) U_{\mu}^{\dagger}(x+\nu) U_{\nu}^{\dagger}(x)
$$

is a Wilson plaquette variable and takes its values in the group $S O(5)^{3}$. Notice that in our study we have taken as generators of $S O(5)$ the matrices $T^{i j}=\frac{1}{4}\left[\gamma^{i}, \gamma^{j}\right]$ where $\gamma_{i}, i=1 \ldots 5$ are the usual four dimensional Dirac matrices together with the chiral matrix $\gamma_{5}$. This differs from the earlier numerical work reported in [16, 17] which utilized the vector representation of $S O(5)$ and implies that we are actually simulating a lattice theory based on the covering group spin(5). As we will see this will turn out to be rather important.

Notice though that the introduction of a lattice has necessarily broken the coordinate invariance of the theory in the base space. Thus one should expect that quantum corrections will generate additional operators whose structure depends on the existence of this background lattice. Assuming that these operators depend only on the $S O(5)$ curvature there are 3 such terms in addition to the Palatini action which are (marginally) relevant by power counting;

- $\int d^{4} x \epsilon_{\mu \nu \lambda \rho} \operatorname{Tr}\left(F_{\mu \nu} F_{\lambda \rho}\right)$

$$
\text { - } \int d^{4} x \operatorname{Tr}\left(F_{\mu \nu} F_{\mu \nu}\right)
$$

\footnotetext{
${ }^{3}$ For simplicity in this study we have not symmetrized our action to ensure rotational invariance nor have we concerned ourselves at this point with issues of reflection positivity
} 


$$
\text { - } \int d^{4} x \operatorname{Tr}\left(\gamma_{5} F_{\mu \nu} F_{\mu \nu}\right)
$$

The first of these is a topological invariant in the continuum analogous to the instanton number and hence can be neglected. In principle we should include lattice versions of both the second and third terms. In practice we have focused these initial investigations on just the second term. On the lattice we implement it with the usual Wilson plaquette action

$$
S_{W}=\sum_{x} \sum_{\mu<\nu} \operatorname{Tr}\left(2-U_{\mu \nu}-U_{\mu \nu}^{\dagger}\right)
$$

Our choice of this term has clear motivation; it clearly will favor configurations in the large $\kappa$ limit with vanishing $S O(5)$ curvature - a feature which will see shortly is not true of the pure Palatini action. Such configurations are minimally required to achieve a connection to classical gravity. It also has the merit of removing potential lattice doubler modes which will be present in the Palatini action as was first observed in [13].

Of course the presence of a Wilson term is not compatible with coordinate invariance in the base space. It will clearly be very important to test for a restoration of this property in any continuum limit. Nevertheless the important point to realize is that this term is necessarily induced in the lattice theory and to have a hope to obtaining the correct continuum limit we should include it in the bare lattice action and tune its coupling appropriately as the lattice spacing is reduced. The lattice action we consider then has the form

$$
S=\kappa\left(\alpha S_{W}+(1-\alpha) S_{P}\right)
$$

which allows us to interpolate between the pure Palatini action defined by $\alpha=0$ and pure Wilson action when $\alpha=1$.

Finally, we need to give a presecription for extracting the various $S O(4)$ components of the connection and curvature from the basic $S O(5)$ variables in the theory. We used the simple expressions for the vierbein and torsion

$$
\begin{aligned}
e_{\mu}^{i} & =\operatorname{Tr}\left[T^{5 i} U_{\mu}\right] \\
T_{\mu \nu}^{i} & =\operatorname{Tr}\left[T^{5 i} \frac{1}{2}\left(U_{\mu \nu}-U_{\nu \mu}\right)\right] \quad i=1, \ldots 4
\end{aligned}
$$

while the $S O(4)$ components of the curvature $\mathcal{R}_{\mu \nu}=R_{\mu \nu}-e_{[\mu} e_{\nu]}$ are given by

$$
\mathcal{R}_{\mu \nu}^{i j}=\operatorname{Tr}\left[T^{i j} \frac{1}{2}\left(U_{\mu \nu}-U_{\nu \mu}\right)\right] \quad i=1 \ldots 4
$$

As in the previous studies [17, 16] we have assumed an $S O(5)$ invariant Haar measure on the group in the path integral defining the quantum theory. We have employed a standard metropolis algorithm to perform the Monte Carlo simulation. 


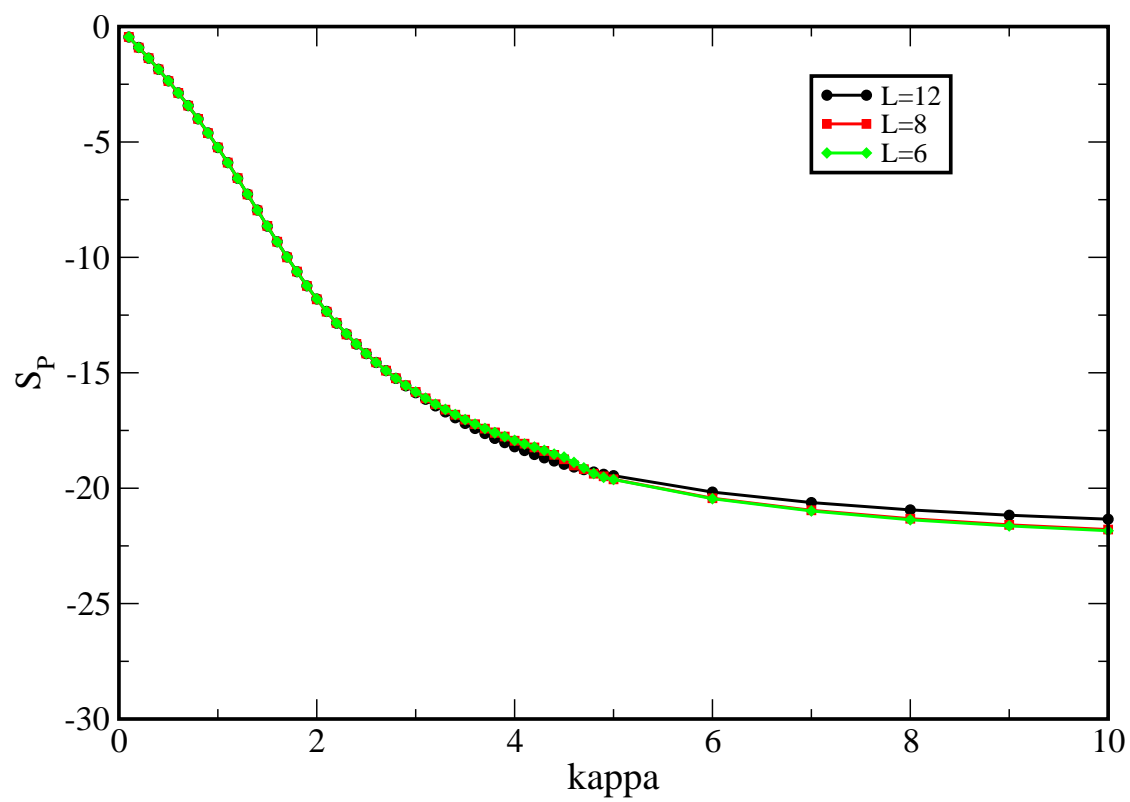

Figure 1: Expectation value of Palatini action for $\alpha=0$ vs coupling $\kappa$

\section{Numerical results}

\subsection{Pure Palatini}

In fig. 1 we show the expectation value of the pure Palatini action $(\alpha=0)$ as a function of the coupling $\kappa$ for a sequence of three lattice sizes $L=6,8,12$. One sees that the action approaches its lower kinematic bound of $S_{P}=-24$ (which reflects the number of non-zeroes of the $\epsilon$ symbol) independent of $L$ as the weak coupling limit $\kappa \rightarrow \infty$ is taken. This limit is reached when the plaquette variables satisfy the extremality condition

$$
U_{\mu \nu}=-\gamma_{5} \epsilon_{\mu \nu \rho \lambda} U_{\rho \lambda}^{\dagger}
$$

We will see later that pure Palatini configurations satisfying this condition possess a maximal $S O(4)$ curvature and hence cannot be interpreted as corresponding to a smooth spacetime. Presumably this reflects the usual unboundedness problem of Euclidean quantum gravity. Furthermore, we observe that the expectation value of the vierbein approaches zero as $\kappa \rightarrow \infty$ as can be seen in fig. 2 in which $\operatorname{Tr}(g)=\sum_{\mu, i} e_{\mu}^{i} e_{\mu}^{i}$ is plotted as a function of $\kappa$ for the same range of lattice sizes ${ }^{4}$. As emphasized earlier the vanishing of the vierbein implies that the corresponding metric $g_{\mu \nu}$ is zero and removes the possibility of interpreting the classical equations of motion as corresponding to the field equations of a metric theory of gravity.

Before discussing the situation with $\alpha>0$ we first return to an issue of what representation should be used for implementing the local Lorentz invariance. As we have described

\footnotetext{
${ }^{4}$ We absorb the scale $l$ into our lattice vierbein throughout this paper
} 


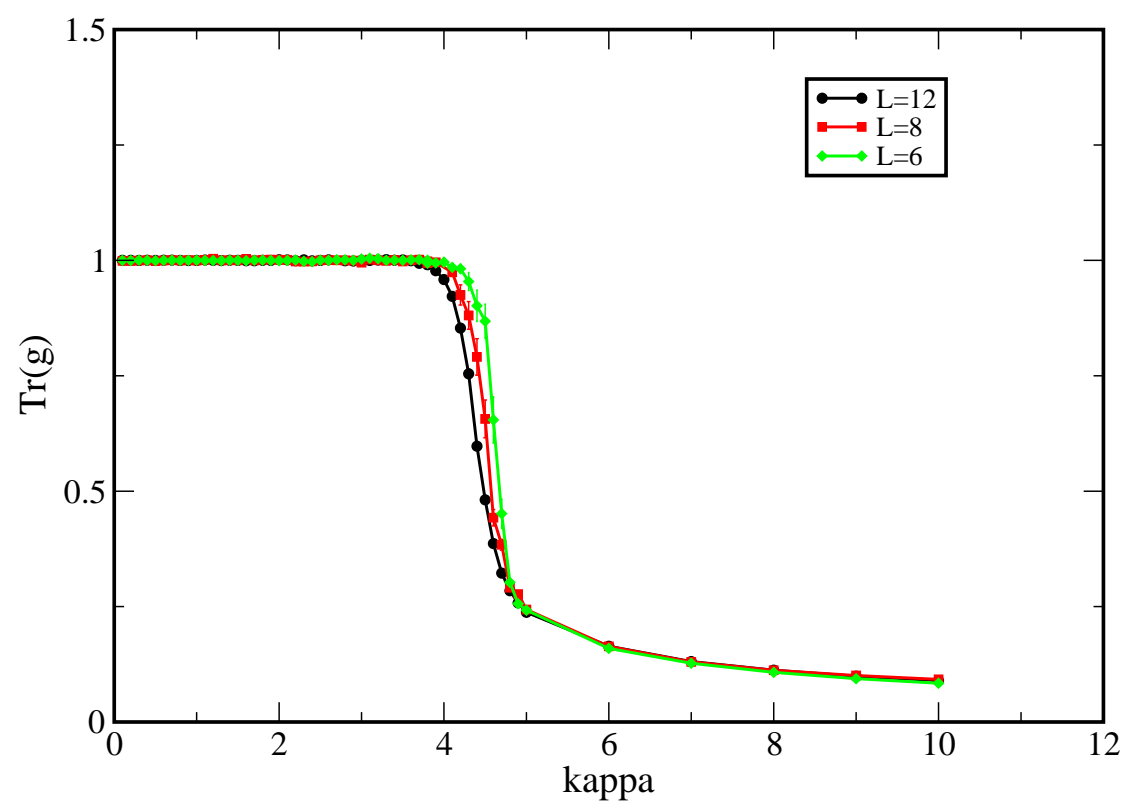

Figure 2: Expectation value of $\operatorname{Tr}(g)$ for $\alpha=0$ vs coupling $\kappa$

in the last section, all the results shown in this paper have been obtained using the four dimensional representation of $\operatorname{spin}(5)$ given in terms of commutators of four dimensional Dirac matrices. As one can see in the previous two plots this choice generates a smooth dependence of observables on the coupling $\kappa$. This is quite different to what was seen in the only previous numerical study of this system where a very strong first order phase transition was observed separating strong from weak coupling. ${ }^{5}$ Indeed the observed hysteresis effects were so severe in the latter case that it was hard to even thermalize the lattices in the weak coupling phase. This earlier study utilized the fundamental or vector representation of $S O(5)$. This observed disparity in the phase structure of these two lattice theories depending on choice of representation is seen even in the pure Wilson theory $(\alpha=1)$ as can be seen in fig. 3 which shows the average action plotted as a function of $\kappa$ for the two representations While the two representations agree at weak coupling as they must since they possess the same Lie algebra, they differ at strong coupling and the lattice action employing the fundamental representation of $S O(5)$ suffers a strong first order transition for $\kappa \sim 0.75$ which is completely absent in the $\operatorname{spin}(5)$ representation. This is analogous to the situation for the lattice theories of $S O(3)$ and $S U(2)$ - the former exhibiting a first order bulk phase transition separating weak from strong coupling while the latter does not. Indeed, the analogy is even stronger since $S U(2) \equiv \operatorname{spin}(3)$ which leads us to conjecture that while the $S O(N)$ groups have first order bulk transitions their covering groups $\operatorname{spin}(N)$ do not. Certainly from a practical point of view the use of the spinor representation is clearly superior to that of the vector representation.

\footnotetext{
${ }^{5}$ One needs to rescale $\kappa$ in this work by 16 to compare the two couplings in these simulations 17,16
} 


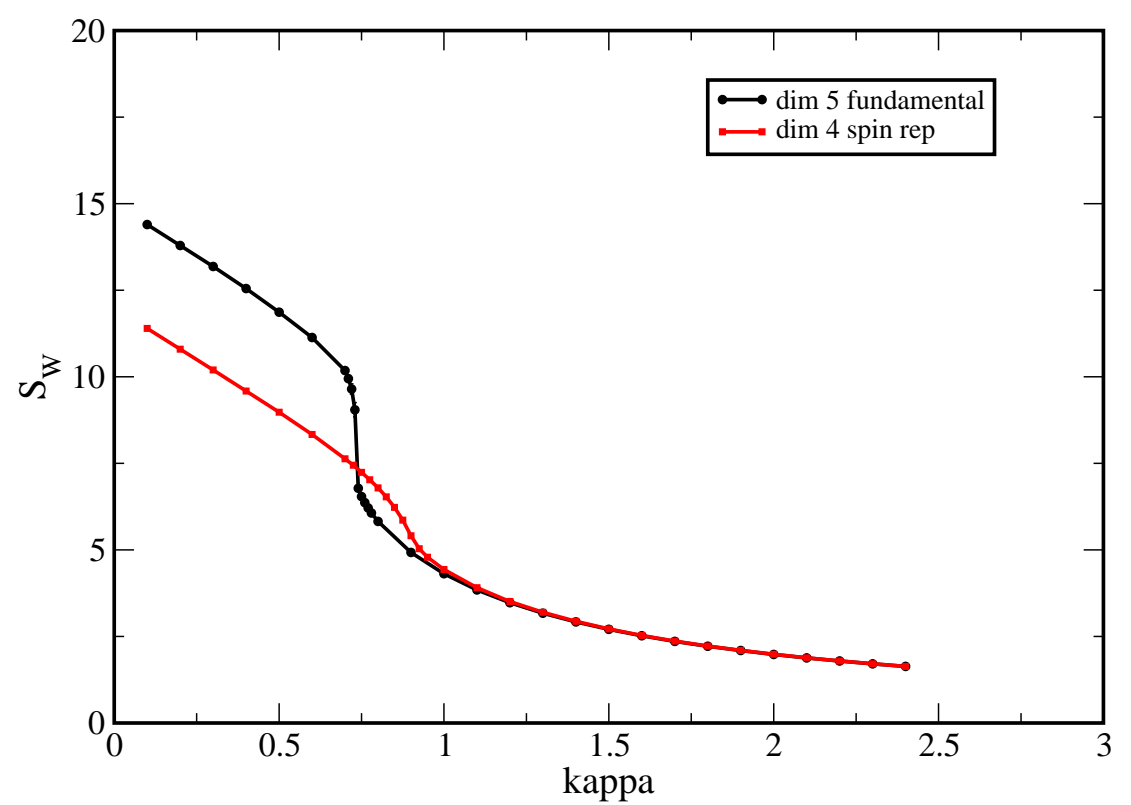

Figure 3: Comparison of vector and spinor representations for $S O(5)$ Wilson action

\subsection{Palatini plus Wilson}

In this section we shows results obtained in the model with $\alpha>0$. First consider the expectation value of the Palatini action in such models. Fig. 1 1 shows this for four different values of the coupling $\alpha=0.0,0.25,0.5,0.75$ for a fixed $8^{4}$ lattice as a function of $\kappa$. Clearly the curves fall into 2 classes; for small $\alpha$ the expectation value of the action approaches the kinematic boundary corresponding to maximal (negative) curvature while for larger $\alpha$ they approach vanishing action in the weak coupling limit. A similar contrasting behavior is seen in the plot of the vierbein as revealed in fig. 5. At small $\alpha$ the vierbein is driven to zero for large $\kappa$ as for pure Palatini while for $\alpha$ above some critical value $\alpha_{T}$ the vierbein remains non-zero in the weak coupling limit. Thus it appears that a sufficiently large coupling to the Wilson operator stabilizes the vierbein. However this is not enough - we also require a zero torsion condition and a small curvature. We now turn to these other observables. Fig. 6 shows a plot of $\mathcal{R}_{\mu \nu}^{i j} \mathcal{R}_{\mu \nu}^{i j}$ and $T_{\mu \nu}^{i} T_{\mu \nu}^{i}$ for the same values of $\alpha$ as a function of the coupling $\kappa$. For small $\alpha$ the expectation values are driven to values similar to the case for pure Palatini the $S O(4)$ curvature attaining near maximal (negative) values as $\kappa \rightarrow \infty^{6}$. However, again, for large values of $\alpha$ both the torsion $T$ and curvature $\mathcal{R}$ approach zero at weak coupling. Indeed, notice that for $\alpha=0.75$ the torsion and curvature are degenerate to within small errors in this limit.

These are quite encouraging results but one might worry that the Palatini action is playing no role at all for large $\alpha$ and the physics is being dominated by just the Wilson term

\footnotetext{
${ }^{6}$ Notice that $\mathcal{R} \rightarrow R$ for $e=0$
} 


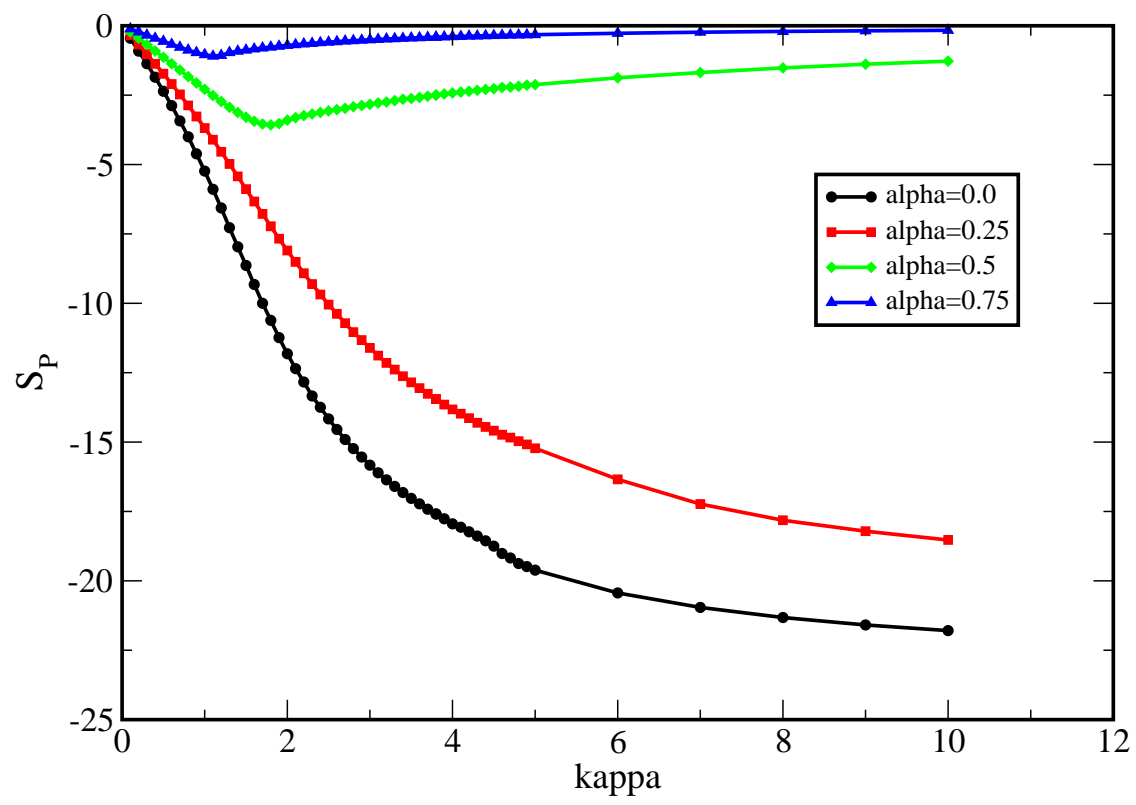

Figure 4: Expectation value of Palatini action vs coupling $\kappa$ for various $\alpha$

- which would invalidate any connection to gravity. To show that this is not the case we have plotted a "self-dual" order parameter given by ${ }^{7}$

$$
P=\frac{\sum_{x} \epsilon_{\mu \nu \lambda \rho} \epsilon^{i j k l} U_{\mu \nu}^{i j} U_{\lambda \rho}^{k l}}{\sum_{x} U_{\mu \nu}^{i j} U_{\mu \nu}^{i j}}
$$

On the extremal configurations given by eqn. 4.1 this attains a value of minus one. This should thus be the case for pure Palatini at large $\kappa$. In contrast it should vanish in the case of the pure Wilson action. In fig. 7 we show the value of this order parameter for the same range of $\alpha$ and $\kappa$ on the $8^{4}$ lattice. Notice that the curves for $\alpha=0.5,0.75$ indicate that order parameter appears to approach a constant for large $\kappa$ at a value which is intermediate between the pure Palatini and Wilson values. We regard this as a piece of evidence disfavoring a system governed solely by the Wilson term.

Up to this point we have concentrated on showing results as a function of $\kappa$ for several discrete values of $\alpha$. However it is also very instructive to show the data as function of $\alpha$ at fixed $\kappa$. For example, the expectation value of the Palatini action is shown in fig. 8 For $\alpha<\alpha_{T}$ with $\alpha_{T}=0.4-0.5$ it is clear that the Palatini action flows to its kinematic lower limit as $\kappa \rightarrow \infty$ independent of the value of $\alpha$ within this range while for $\alpha>\alpha_{T}$ the trend is reversed and the action goes to zero for large enough $\kappa$. This suggests two different ground

\footnotetext{
${ }^{7}$ This is analogous to the self-dual order parameter introduuced in 16 although in that case the duality operation involves only the internal space
} 


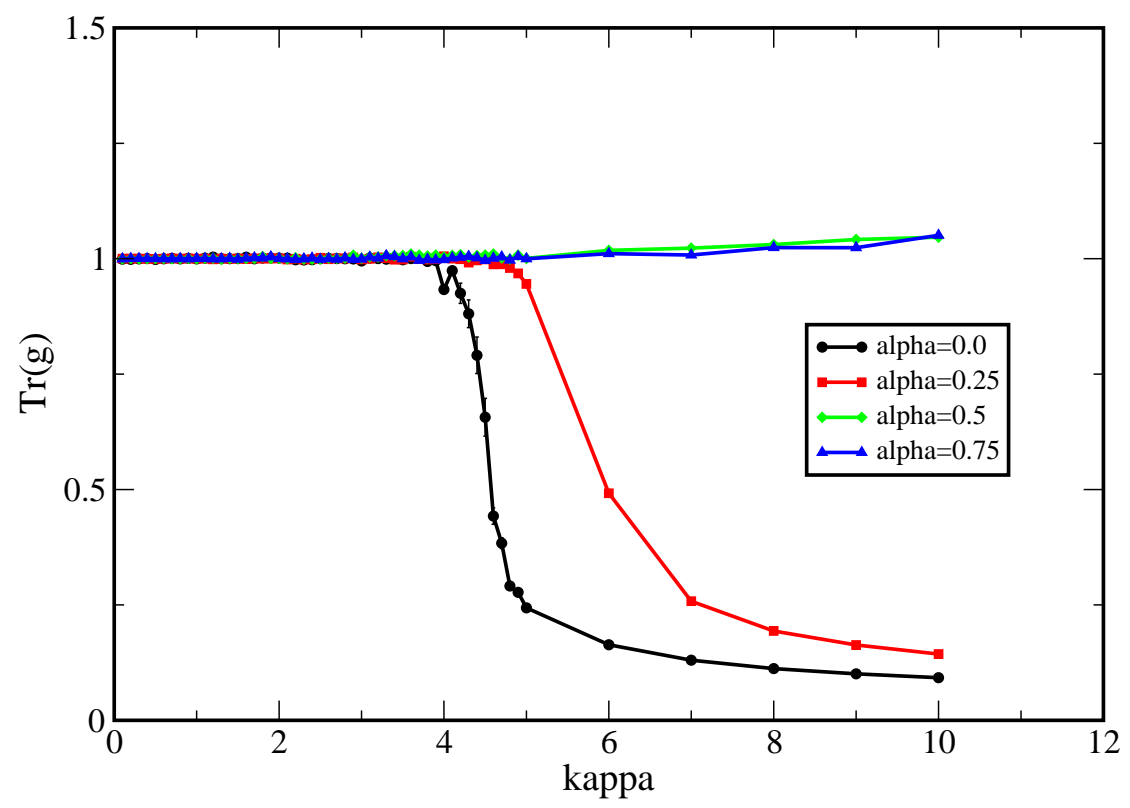

Figure 5: Expectation value of $\operatorname{Tr}(g)$ vs coupling $\kappa$ for various $\alpha$

states are available to the system at least for large enough $\kappa$ whose domain of attraction is determined by the coupling $\alpha$. For $\alpha<\alpha_{T}$ the system at weak coupling is governed by the pure Palatini action while for $\alpha>\alpha_{T}$ the system flows to a ground state corresponding to vanishing $S O(5)$ curvature. In the limit $\alpha \rightarrow 1$ this is just the usual Wilson vacuum. To contrast with this behavior notice that for small $\kappa$ coupling eg $\kappa=0.5$, the value of the action depends only very weakly on the associated coupling $\alpha$ and a system seems to exist in just one phase. Of course one expects that the ground state of the system for small $\kappa$ is dominated by lattice artifacts so this latter phase is ultimately uninteresting.

This cross-over between two different ground states for large $\kappa$ is clearly seen by looking at the torsion and curvature as a function of $\alpha$. A plot (fig. 9) showing $T$ and $\mathcal{R}$ for $\kappa=20.0$ as a function of $\alpha$ confirms the importance of this threshold coupling $\alpha_{T}$ As $\alpha$ increases the curvature drops towards to zero rapidly merging with the torsion for $\alpha>\alpha_{T} \sim 0.5$.

A similar picture is revealed by looking at the plot of the order parameter $P$ as shown in fig. 10. It appears that the order parameter equals minus one for all $\alpha<\alpha_{T}$ as $\kappa \rightarrow \infty$ but then rises towards zero as $\alpha$ varies in the range $\alpha>\alpha_{T}$. Notice also that there is little $\kappa$ dependence in the curves for $\alpha>\alpha_{T}$ - the curves for different $\kappa$ rapidly approach fixed well-defined envelope in this region of parameter space. Again, let us emphasize that the small $\kappa$ data doesn't fit this picture - there is no evidence for a threshold value of $\alpha$ in the $\kappa=0.5$ curve. Thus the phase diagram seems to contain a single strong coupling phase but the possibility of two dramatically different phases at large $\kappa$ coupling. It is unclear from 


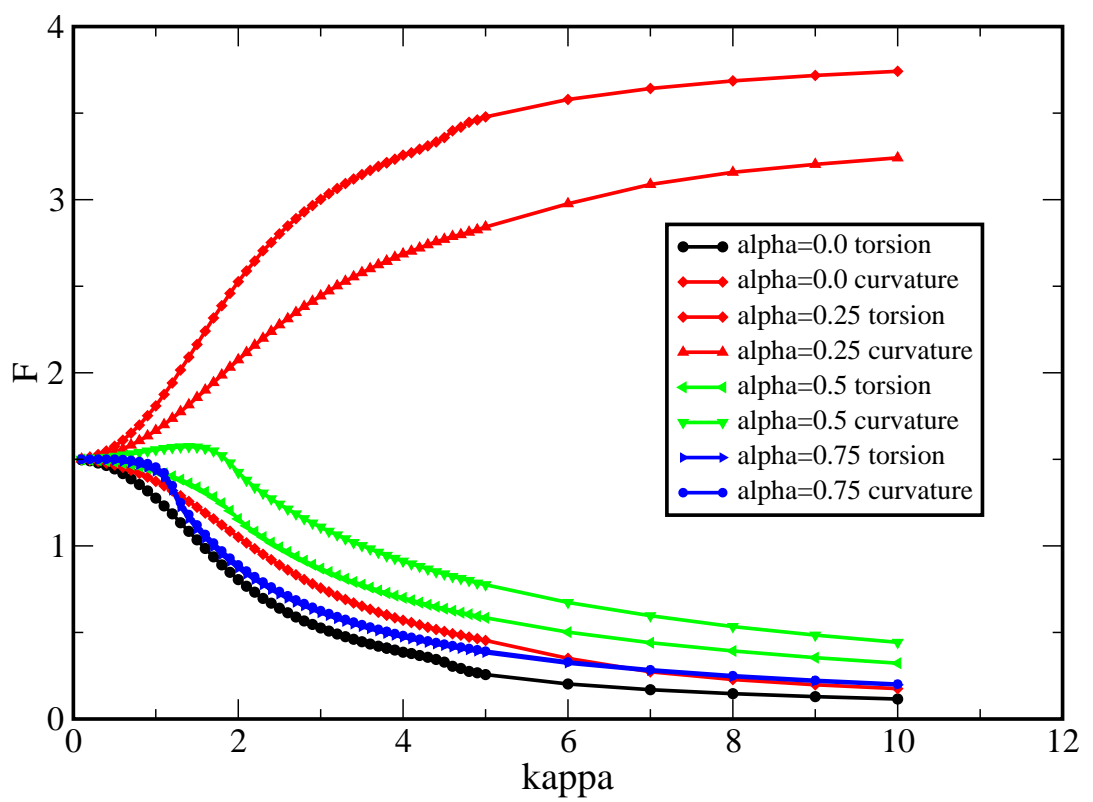

Figure 6: Curvature and torsion components of the $S O(5)$ field strength vs $\kappa$

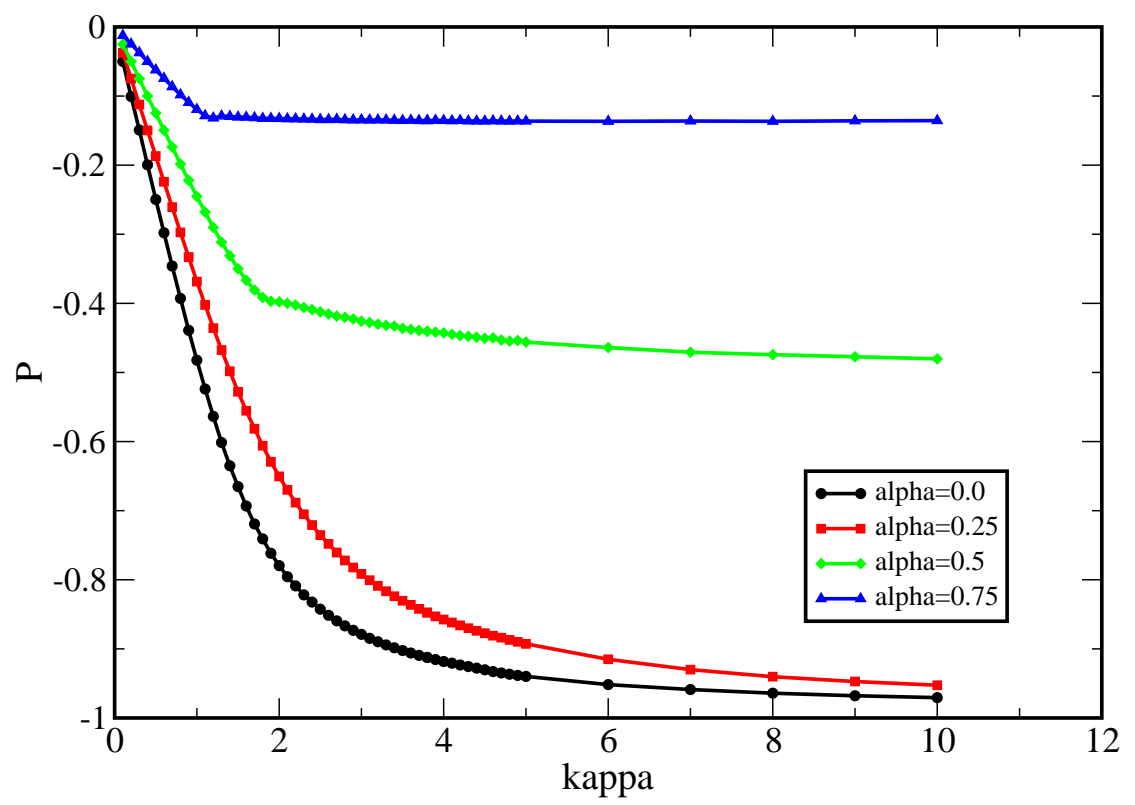

Figure 7: Order parameter $P$ as a function of $\kappa$ for several $\alpha$

the data whether there is a discontinuity or not for $\alpha \rightarrow \alpha_{T}$. One hint favoring a true phase transition at $\alpha_{T}$ can be obtained by looking at the local volume element $\epsilon^{i j k l} \epsilon_{\mu \nu \lambda \rho} e_{\mu}^{i} e_{\nu}^{j} e_{\lambda}^{k} e_{\rho}^{l}$. 


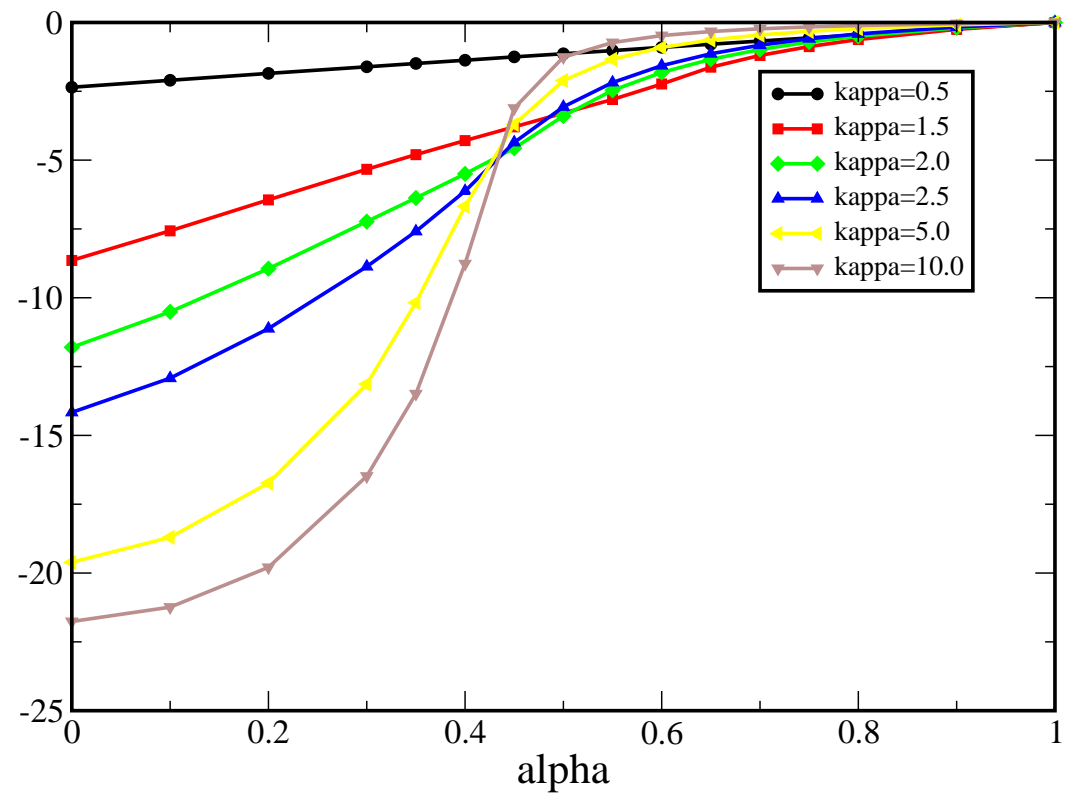

Figure 8: Expectation value of Palatini action as function of $\alpha$ for several $\kappa$

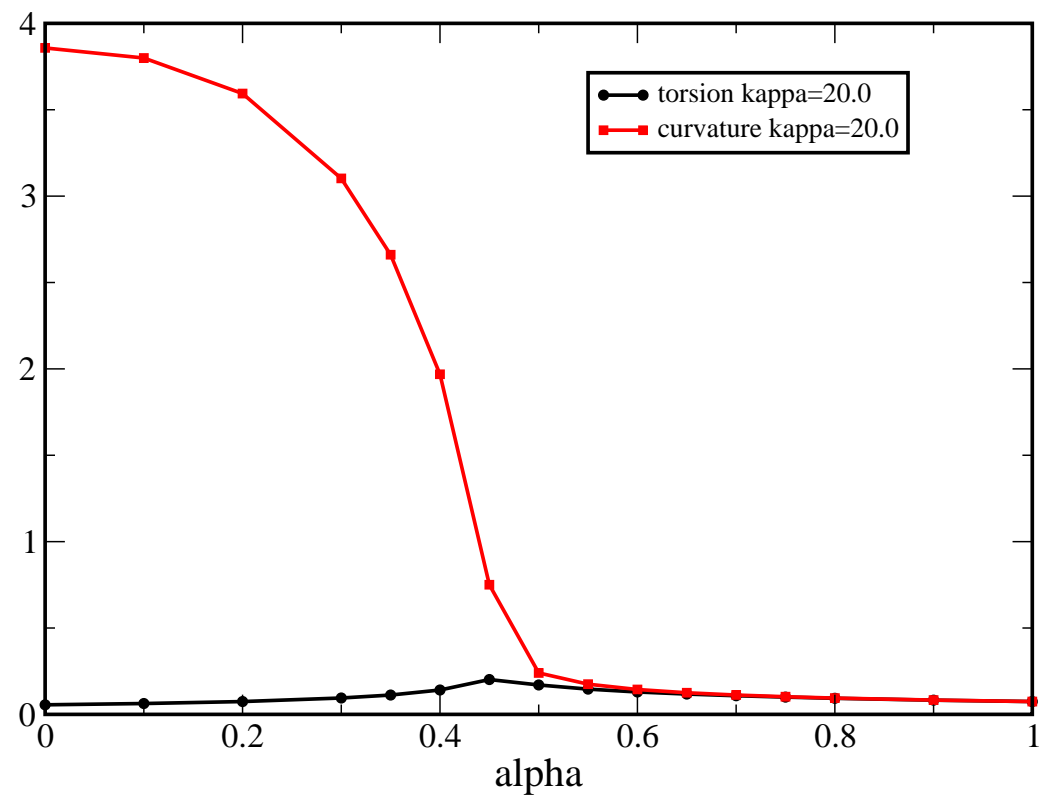

Figure 9: Curvature and torsion as a function of $\alpha$ for $\kappa=20.0$

We can extract a gauge invariant measure for this on the lattice by extracting an expression 


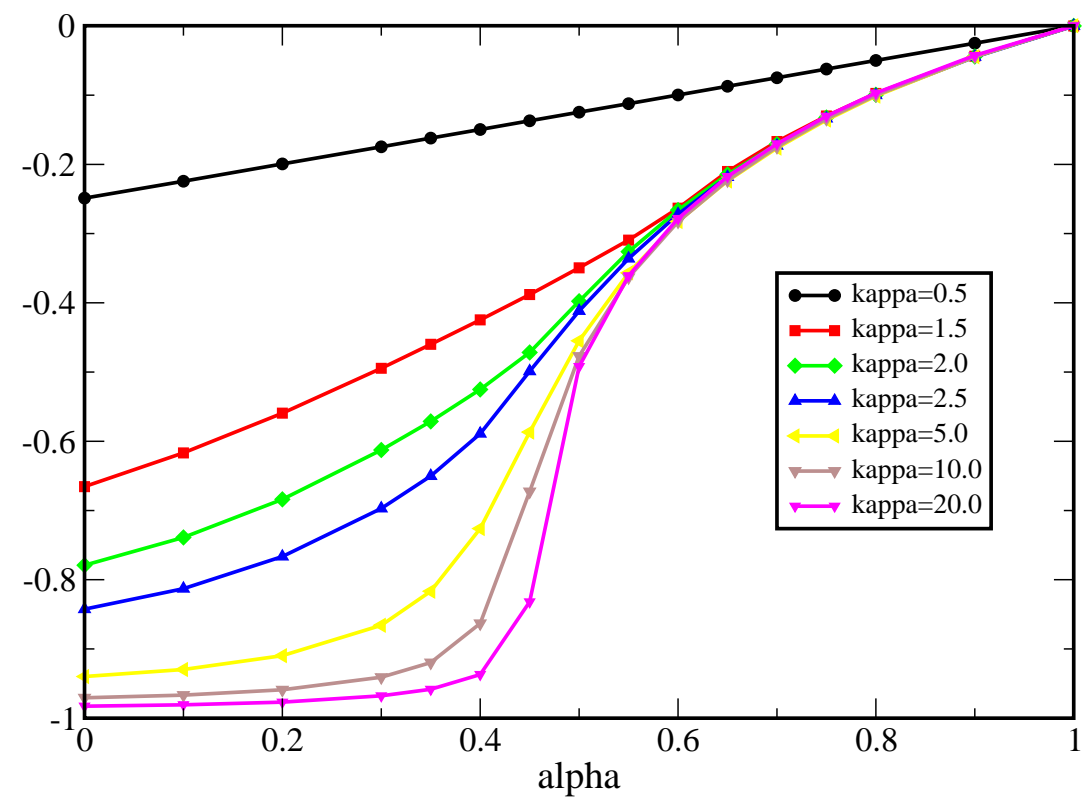

Figure 10: Order parameter as a function of $\alpha$ for several $\kappa$

for a matrix valued representation of the vierbein at a site:

$$
e_{\mu}(x)=\gamma_{5} U_{\mu}(x) \gamma_{5} U_{\mu}^{\dagger}(x)-I
$$

which leads to an expression for the gauge invariant volume element

$$
\sqrt{g}=\epsilon_{\mu \nu \lambda \rho} \operatorname{Tr}\left(e_{\mu} e_{\nu} e_{\lambda} e_{\rho}\right)
$$

The absolute value of this quantity is shown in fig. 11 for $\kappa=20.0$ and $L=8$ as a function of $\alpha$. For small $\alpha$ the vierbein vanishes and so does the volume element while for $\alpha>\alpha_{T}$ it becomes constant. However, in the vicinity of $\alpha_{T}$ we see a sharp spike corresponding to a large local volume element in lattice units.

\section{Discussion}

We have simulated a Euclidean model for quantum gravity based on discretization of the Palatini action including a cosmological constant term and supplemented by a Wilson term. Unlike a previous study of the pure Palatini action we have employed the spinor representation of the spin(5) covering group to define the lattice action. This is faster to implement and appears to avoid some of the strong lattice artifacts seen in earlier studies of this action.

We show that the pure Palatini lattice theory is sick suffering from a local curvature which attains the maximal (negative) value consistent with the compact gauge symmetry, a vanishing vierbein and lattice doublers. 


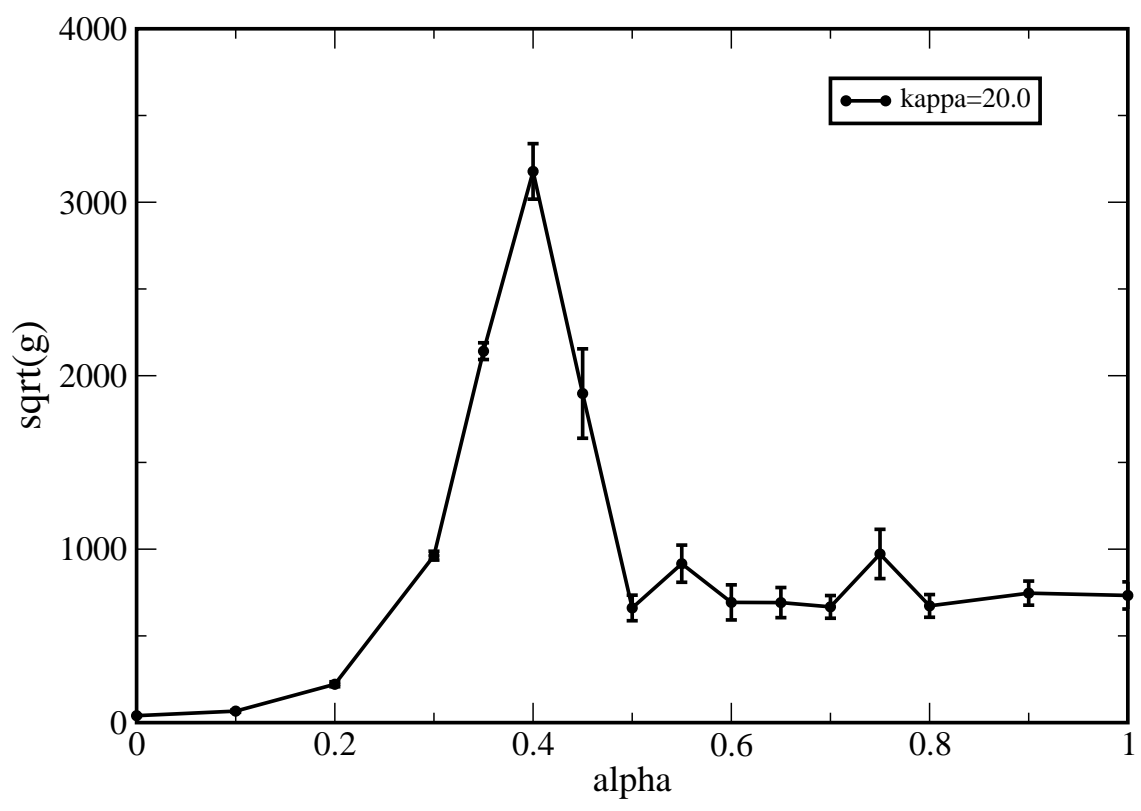

Figure 11: Absolute value of the volume element vs $\alpha$ for $\kappa=20.0$

We argue that the Wilson operator serves both to regulate all of these problems and furthermore will necessarily be induced at the quantum level if it is not present in the bare lattice action. In the expanded parameter space determined by the Palatini and Wilson couplings we find evidence for two new ground states for sufficiently weak coupling (large $\kappa$ ) - one corresponding to pure Palatini and suffering from all the old problems and a second in which the vierbein is non-zero, and the torsion and curvature are small. These two vacua can be distinguished by a self-dual order parameter and can be accessed by tuning the coupling $\alpha$ which controls the mixing with the Wilson term.

It appears that for $\alpha$ couplings close to some threshold value $\alpha_{T}$ the new vacuum is not just that of the pure Wilson action but retains a memory of the Palatini term as revealed by the self-dual order parameter acquiring a non-trivial value there. It remains to be seen whether this threshold value $\alpha_{T}$ can be thought of as a true critical value and if so whether this critical value corresponds to a continuous or discontinuous phase transition. The latter issue is of course a crucial issue to address in the context of obtaining a non-trivial continuum limit.

\section{Acknowledgments}

SMC and DF are supported in part by DOE grant DE-FG02-85ER40237. The simulations were carried out using USQCD resources at Fermilab. 


\section{References}

[1] J. F. Donoghue, General relativity as an effective field theory: The leading quantum corrections, Phys. Rev. D50 (1994) 3874-3888, gr-qc/9405057.

[2] Y. Ne'eman and T. Regge Phys. Lett. B74 (1978) 54.

[3] S. Catterall, J. B. Kogut, and R. Renken, Phase structure of four-dimensional simplicial quantum gravity, Phys. Lett. B328 (1994) 277-283, hep-lat/9401026.

[4] J. Ambjorn and J. Jurkiewicz, Four-dimensional simplicial quantum gravity, Phys. Lett. B278 (1992) 42-50.

[5] B. V. de Bakker and J. Smit, Curvature and scaling in 4-d dynamical triangulation, Nucl. Phys. B439 (1995) 239-258, hep-lat/9407014.

[6] M. E. Agishtein and A. A. Migdal, Critical behavior of dynamically triangulated quantum gravity in four-dimensions, Nucl. Phys. B385 (1992) 395-412, hep-lat/9204004.

[7] J. Ambjorn, A. Gorlich, J. Jurkiewicz, and R. Loll, The Nonperturbative Quantum de Sitter Universe, Phys. Rev. D78 (2008) 063544, 0807.4481.

[8] J. Ambjorn, J. Jurkiewicz, and R. Loll, The Self-Organized de Sitter Universe, Int. J. Mod. Phys. D17 (2009) 2515-2520, 0806.0397.

[9] J. Ambjorn, A. Gorlich, J. Jurkiewicz, and R. Loll, Planckian Birth of the Quantum de Sitter Universe, Phys. Rev. Lett. 100 (2008) 091304, 0712.2485.

[10] L. Smolin, QUANTUM GRAVITY ON A LATTICE, Nucl. Phys. B148 (1979) 333.

[11] P. Menotti and A. Pelissetto, GAUGE INVARIANCE AND FUNCTIONAL INTEGRATION MEASURE IN LATTICE GRAVITY, Nucl. Phys. B288 (1987) 813.

[12] P. Menotti and A. Pelissetto, POINCARE, DE SITTER AND CONFORMAL GRAVITY ON THE LATTICE, Phys. Rev. D35 (1987) 1194.

[13] P. Menotti and A. Pelissetto, REFLECTION POSITIVITY AND GRAVITON DOUBLING IN EUCLIDEAN LATTICE GRAVITY, Ann. Phys. 170 (1986) 287.

[14] S. Caracciolo, P. Menotti, and A. Pelissetto, LATTICE SUPERGRAVITY AND GRAVITON GRAVITINO DOUBLING, Nucl. Phys. B296 (1988) 868.

[15] S. Caracciolo and A. Pelissetto, ANALYSIS OF THE CRITICAL BEHAVIOR OF DE SITTER QUANTUM GRAVITY ON A HYPERCUBIC LATTICE, Phys. Lett. B193 (1987) 237.

[16] S. Caracciolo and A. Pelissetto, PHASES AND TOPOLOGICAL STRUCTURES OF DE SITTER LATTICE GRAVITY, Nucl. Phys. B299 (1988) 693.

[17] S. Caracciolo and A. Pelissetto, A NUMERICAL INVESTIGATION ABOUT QUANTUM MEASURE IN LATTICE GRAVITY, Phys. Lett. B207 (1988) 468-470.

[18] S. W. MacDowell and F. Mansouri, Unified Geometric Theory of Gravity and Supergravity, Phys. Rev. Lett. 38 (1977) 739. 